\title{
Review of: "Association patterns of cannabis abuse and dependence with risk of problematic non- substance-related dysregulated and addictive behaviors"
}

\author{
Migle Kaminskaite ${ }^{1}$ \\ 1 Lithuanian University of Health Sciences
}

Potential competing interests: The author(s) declared that no potential competing interests exist.

1.C.Perales with coleagues evaluates the co-occurrence of canabis abuse/dependence and addiction-similar behaviours in their article "Association patterns of cannabis abuse and dependence with risk of problematic non-substance-related dysregulated and addictive behaviors". This research could provide deeper insight into the pathogenesis of both substance use disorders and addiction-like behaviours. Besides the scientifical value, the research may have a practical one, as a better unterstanding of addictions might lead to better methods of prevention and intervention. More than this, the authors take into account the possible gender differences in their analyses and demonstrate the divergent associations with addiction-like behaviours and cannabis abuse/dependence for males and females. The gender-specific analyses for addictions and addiction-like pathologies are strongly encouraged with respect to the different patterns of addiction development and possible causes for males and females.

The article is well writen and comprehensible. However, I think that such expresions as "bona fide" might be not intuitively understandable for some readers. Please change this. Also, please explain the abbreviations when using them for the first time (the part of "The present study“, first paragraph: CAST, SDS, MultiCAGE, second paragraph: CAGE). While these are well known to someone who is working in the same field, some of your readers could be not so experienced.

The authors state that both substance-use-related disorders and addiction-like behaviour pathologies are from the spectrum of externalizing behaviour pathologies, and because of this the authors suggest the existence of similarities in ethiology of substance and behavioural addictions. However, while this is true in most of the cases, I would not state that all addictions and similar pathologies are only on the externalizing end in spectrum. While the evidence for internalizing spectrum pathologies is not so clear, they also have been demonstrated to impact the risk of addiction-like conditions [1] [2]. Although the co-occurrence of substance abuse and behavioural addictions with internalizing pathologies does not deny the theory of similar pathologies, but instead augments it with respect to possible different pathways, vulnearability associated phenotypes and motivational mechanisms.

The methods are accurate and elegant. The authors did their best to provide a representative sample and included the possibility of sample not being representative enough in the section of limitations. The authors 
chose an appropriate instrument to measure addiction-like behaviours. Cannabis abuse/dependence was measured by both CAST and SUD scales in the study. Here an explanation of the choice to use both scales and detailing the differences of both scales and their measures would be needed. Moreover, there are some differences in the results (e.g. different impact of interactions with gender by SUD and CAST on the risk of compulsive buying and gambling). These results need some explanation and maybe providing differences of the CAST and SUD scales in the methods section would make it easier to explain differences of results.

The cut-off values of the CAST and SUD are provided in the methods section. However, the authors used the total score of CAST/SUD in analyses but did not stratified the groups according to the degree of the risk. Please, explain that choice.

For the statistical analysis authors have choosen the mixed-effect regression. I find this method suitable, however, it is not the most often used method in this type of studies, so the article would benefit if the authors would add a few words about their choice. Also, I have some concerns about presentation of the results. In particular, tables 2 and 3 don't seem to be self-explanatory. Please provide the explanations for abbreviations below the table, so that the reader wouldn't have to look for them through all the text. Please provide the reference groups - e.g., I do not think that the gender itself had an effect to the higher risk, but it was the male gender compared to females?

Did the CASTXGender interaction, which was non-significant, impact model-fit? I do not think so? Why is this interaction in grey? Should it be really included in the model? Also I assume the CASTxGender interaction for risk of compulsive buying was considered for family-wise correction, although it is not in grey?

Why don't you add a $95 \% \mathrm{Cl}$.as some readers prefer this to $\mathrm{p}$ value? Some readers might prefer this as it is more intuitive to see if the Confidence interval was wide or narrow when estimating effect sizes or IRR. Overall, I think that the study is valuable and the article is suitable for publishing, but would benefit from some minor corrections that I have mentioned.

1. Richard J, Fletcher É, Boutin S, Derevensky J, Temcheff C. Conduct problems and depressive symptoms in association with problem gambling and gaming: A systematic review. J Behav Addict. 2020;9: 497-533. doi:10.1556/2006.2020.00045

2. Laier C, Wegmann E, Brand M. Personality and Cognition in Gamers: Avoidance Expectancies Mediate the Relationship Between Maladaptive Personality Traits and Symptoms of Internet-Gaming Disorder. Front Psychiatry. 2018;9. doi:10.3389/fpsyt.2018.00304 